\title{
Prevalência e fatores de risco para retinopatia diabética em pacientes diabéticos atendidos por demanda espontânea: um estudo transversal
}

Prevalence and risk factors of diabetic retinopathy in patients with diabetes seen by unscheduled demand: a cross-sectional study

Fernanda Mendonça Galvão1 (1), Yael Porto Silva ${ }^{1}$ (D), Mateus Inácio Lemes de Resende ${ }^{2}$ (1) Frederico Romano Barbosa ${ }^{2} \mathbb{1}$, Thiago Alves Martins $\left.{ }^{2} \mathbb{(}\right)$, Luciana Barbosa Carneiro ${ }^{2}$ (1)

Descritores:

Diabetes mellitus; Retinopatia diabética; Epidemiologia; Fatores de risco; políticas públicas de saúde

Keywords: Diabetes mellitus; Diabetic retinopathy; Epidemiology; Risk factors; Public health policy

Submetido: 20/11/2020

Aceito:

$1 / 4 / 2021$

Autor correspondente: ernanda Mendonça Galvão Avenida, 355-447 - Setor Leste Universitário CEP: 74605-020 - Goiânia, GO, Brasil E-mail: nanda.mdgv@gmail.com

Instituição de realização do trabalho: Hospital Fundação Banco de Olhos de Goiás, Goiânia, GO, Brasil.

Fonte de auxílio à pesquisa: não financiado.

Conflitos de interesse: os autores declaram que não há conflitos de interesses.

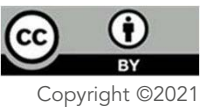

\section{RESUMO}

Objetivo: Estimar a prevalência da retinopatia diabética em pacientes diabéticos de uma capital brasileira e correlacioná-la com fatores de risco presentes na população estudada.

Métodos: Estudo observacional transversal, realizado a partir do relatório de atendimentos prestados em mutirão ocorrido em 2018. O relatório foi preenchido pelos médicos oftalmologistas durante a campanha, com informações referentes a sexo do paciente, idade, classificação do diabetes mellitus, tempo de doença, uso de insulina, índice de massa corporal, hábitos de vida (tabagismo e atividade física) e história de hipertensão arterial sistêmica, dislipidemia, infarto agudo do miocárdio e acidente vascular cerebral, além de exame clínico oftalmológico realizado na ação.

Resultados: Dentre os 219 participantes do estudo, a prevalência da retinopatia diabética foi de $31,96 \%$. As variáveis que se apresentaram como fator de risco com significância estatística foram sexo masculino, idade de 51 a 70 anos, mais de 10 anos de diabetes mellitus, insulinoterapia, índice de massa corporal $\geq 40 \mathrm{~kg} / \mathrm{m}^{2}$ e história prévia de infarto agudo do miocárdio. Atividade física mostrou-se como fator protetor significativo.

Conclusão: Estudos populacionais ao longo dos anos comprovaram a variabilidade geográfica na prevalência da retinopatia diabética justificada pela diferente exposição aos fatores de risco. Dentro de tal conjuntura, ressalta-se o quão fundamental é o conhecimento das características regionais, de modo a orientar as políticas de saúde pública, permitindo atuar com impacto na redução das estatísticas de cegueira evitável.

\section{ABSTRACT}

Objective: To estimate the prevalence of diabetic retinopathy in patients with diabetes, from a Brazilian capital city, and to correlate with the risk factors present in the studied population.

Methods: A cross-sectional observational study, based on the report of care provided by a campaign, in 2018. The report was filled out by ophthalmologists during the joint effort, with information on patient's gender, age, classification of diabetes mellitus, duration of illness, use of insulin, body mass index, lifestyle (smoking and physical activity), history of hypertension, dyslipidemia, myocardial infarction, stroke, and the clinical ophthalmic examination.

Results: Among the 219 study participants, the prevalence of diabetic retinopathy was $31.96 \%$. The variables considered risk factors with statistical significance were male sex, age 51-70 years, diabetes mellitus for over 10 years, insulin therapy, body mass index $\geq 40 \mathrm{~kg} / \mathrm{m}^{2}$, and previous history of myocardial infarction. The physical activity proved to be a significant protective factor.

Conclusion: Over the years, population studies have proven the geographical variability in prevalence of diabetic retinopathy justified by different exposure to risk factors. Therefore, knowledge of regional characteristics is crucial and emphasized in the text, since it can guide public health policies, aiming to have an impact on reduction of preventable blindness statistics. 


\section{INTRODUÇÃO}

O diabetes mellitus (DM) é uma das principais doenças crônicas que acomete populações em todo o mundo. Segundo a Sociedade Brasileira de Diabetes (SBD), a população de pessoas com diabetes no Brasil é estimada em 13 milhões, e o país ocupa a quarta posição no ranking mundial. ${ }^{(1)}$ Entre 2006 e 2017, a prevalência passou de $5,5 \%$ para $8,9 \%$, com percentual elevado de desconhecimento do diagnóstico. De acordo com dados da Vigilância de Fatores de Risco e Proteção para Doenças Crônicas por Inquérito Telefônico (Vigitel), houve aumento de casos de $54 \%$ entre os homens e de $28 \%$ entre as mulheres, destacando-se o incremento de $24 \%$ nos pacientes com mais de 65 anos de idade. ${ }^{(2)}$

O desconhecimento do diagnóstico traz como consequências complicações micro e macrovasculares, frequentemente em estágios adiantados, o que contribui para aumentar o já elevado custo do DM no Brasil: US\$97 bilhões em estimativas mais conservadoras ou até US\$123 bilhões, em um pior cenário, em 2030.(1) Dentre essas complicações, encontra-se a retinopatia diabética (RD), uma das principais causas de perda visual irreversível no mundo e considerada a maior causa de cegueira na população entre 16 e 64 anos. ${ }^{(3)}$ Os custos da perda de produção são cinco vezes o custo com os cuidados de saúde diretos, de acordo com estimativas provenientes de 25 países latino-americanos. ${ }^{(4)}$

O desenvolvimento e a progressão da RD podem estar associados a fatores de risco modificáveis e não modificáveis. Os primeiros incluem hiperglicemia crônica, hipertensão, dislipidemia e obesidade. Por outro lado, a duração do diabetes, a puberdade e a gravidez são fatores de risco não modificáveis. ${ }^{(3)}$ Entretanto, estudos populacionais mundiais revelaram a variabilidade geográfica na prevalência da RD pela diferente exposição aos fatores de risco. ${ }^{(5)}$

Nesse cenário, é de fundamental importância o conhecimento das características regionais para orientar as políticas públicas e atuar com impacto na redução das estatísticas de cegueira evitável.

O objetivo deste estudo foi estimar a prevalência da RD em uma capital brasileira e correlacioná-la com os fatores de risco presentes na população estudada.

\section{MÉTODOS}

Trata-se de estudo observacional transversal, realizado a partir do relatório de atendimentos prestados em um mutirão voltado para pacientes com diabetes, por demanda espontânea, ocorrido em Goiânia (GO), no ano 2018, promovido pelo Hospital Fundação Banco de Olhos de Goiás, referência oftalmológica da Região Centro-Oeste brasileira. O relatório foi preenchido pelos médicos oftalmologistas durante a campanha, com informações referentes a sexo do paciente; idade; classificação do DM (tipo 1 ou 2); tempo de doença; uso de insulina; índice de massa corporal (IMC); hábitos de vida (tabagismo e atividade física); história de hipertensão arterial sistêmica (HAS), dislipidemia (DLP), infarto agudo do miocárdio (IAM) e acidente vascular cerebral (AVC) e do exame clínico oftalmológico realizado na ação.

Dados de histórico médico e de hábitos de vida foram confirmados por autorrelato e pelas medicações em uso. O IMC foi calculado a partir de dados coletados em balança calibrada e fita métrica no local da campanha. A classificação do IMC seguiu as normas das Diretrizes Brasileiras de Obesidade: ${ }^{(6)}$ entre 18,5 e $24,9 \mathrm{~kg} / \mathrm{m}^{2}$, o peso foi considerado normal; de 25 a 29,9kg/m², sobrepeso; 30 a 34,9kg/ $\mathrm{m}^{2}$, obesidade grau I; de 35 a $39,9 \mathrm{~kg} / \mathrm{m}^{2}$, obesidade grau II e, por fim, valores acima de $40 \mathrm{~kg} / \mathrm{m}^{2}$ ficam na categoria de obesidade grau III. Pelo exame oftalmológico, os pacientes foram classificados segundo o Early Treatment Diabetic Retinopathy Study (ETDRS) ${ }^{(7)}$ e, posteriormente, agrupados em cinco categorias: sem RD aparente, RD não proliferativa (RDNP) leve a moderada, RDNP grave, RD proliferativa (RDP) e edema macular (EM).

Foram incluídos participante com DM e com todos os dados preenchidos. Foram excluídos aqueles para os quais constava opacidade de meios (impossibilidade de visualização do fundo de olho por oftalmoscopia indireta) e que descreviam infecção ocular contemporânea ou qualquer outra patologia oftalmológica que não a RD.

Os dados coletados foram transferidos para uma planilha eletrônica da Microsoft Excel, para posterior análise bioestatística no software Epi Info ${ }^{\mathrm{TM}}$ 7.2. Taxas de amostragem independentes foram comparadas pelo teste do qui-quadrado. Para demais associações, foi utilizado a razão de prevalência. Valor de p<0,01 foi considerado para indicar significância estatística.

\section{RESULTADOS}

Foram considerados 219 pacientes, dos quais 131 (59,82\%) eram do sexo feminino. Quanto à idade, cinco (2,28\%) pacientes tinham entre zero e 25 anos, 37 (16,89\%) entre 26 e 50 anos, 133 (60,73\%) entre 51 e 70 anos, e 44 (20,09\%) participantes tinham mais de 70 anos de idade. Sobre o tipo de DM, 94,98\% (208) dos pacientes afirmavam ter DM tipo 2 e os demais 5,02\% (11) referiam DM tipo 1. Dentre todos, $98(44,75 \%)$ afirmavam ter conhecimento 
da doença há menos de 5 anos; 51 (23,29\%) de 5 a 10 anos, e $70(31,96 \%)$ há mais de 10 anos. Houve predominância de não insulinodependentes, representando 73,06\% (160) da população estudada.

No que diz respeito ao IMC, 59 (26,94\%) pacientes apresentaram peso adequado; 76 (34,70\%), sobrepeso; 43 (19,63\%), obesidade grau I; 29 (13,24\%), obesidade grau II e 12 (5,48\%), obesidade grau III. Ainda, 48,40\% (106) dos integrantes eram não tabagistas, 45,21\% (99) eram ex-tabagistas e 6,39\% (14) ainda fumavam. Além disso, 99 $(45,21 \%)$ pacientes também referiram realizar atividade física pelo menos três vezes na semana. Quanto aos antecedentes pessoais patológicos, 63,01\% (138) relatava HAS, 43,38\% (95) DLP, 6,39\% (14) IAM e 4,57\% (10) história prévia de AVC. Em relação à prevalência da retinopatia na população estudada, 149 (68,04\%) participantes não apresentavam RD, enquanto 35 (15,98\%) tinham RDNP leve a moderada, 18 (8,22\%) RDNP grave e 17 (7,76\%) RDP. Dentre os 70 pacientes com RD, 21 (30,00\%) eram acometidos por EM, representando 9,59\% da população total.

O sexo masculino demonstrou relevância estatística significativa como fator de risco, apresentando frequência relativa de 53,40\% dentre os pacientes com RD, razão de prevalência de 2,09 com intervalo de confiança (IC) entre 1,49 e 2,93, para p=0,00001. Com relação à idade, o teste do qui-quadrado verificou significância estatística com maior prevalência entre 51 e 70 anos de idade $(\mathrm{p}=0,0073)$.

No presente estudo, o DM tipo 1 não apresentou valores com significância estatística: razão de prevalência de 1,43 (IC 0,93-2,19; p=0,14), apesar de frequência relativa de $52,1 \%$, possivelmente devido à população mais idosa do mutirão. Quanto ao tempo de doença, o teste do qui-quadrado demonstrou significância estatística, com maior prevalência nos pacientes com mais de 10 anos de doença $(\mathrm{p}=0)$. O uso de insulina também se comprovou como fator de risco, com frequência relativa de 56,72\% e razão de prevalência de 1,71 (IC 1,26-2,32; p=0,0016).

No tocante ao IMC, o teste do qui-quadrado revelou significância estatística com maior prevalência nos pacientes com IMC $\geq 40 \mathrm{~kg} / \mathrm{m}^{2}$ (p=0,0002). Diante dos hábitos de vida, apenas a atividade física mostrou-se como fator protetor estatisticamente significativo, com frequência relativa de $36,1 \%$ e razão de prevalência de 0,59 (IC 0,42-0,84; $\mathrm{p}=0,0028)$. O tabagismo obteve frequência relativa de 35,92\% e razão de prevalência de 1,06 (IC 0,76$1,48 ; \mathrm{p}=0,84)$. Por fim, dos antecedentes pessoais patológicos, a história prévia de IAM foi a única a apresentar valores significativos como fator de risco, com frequência relativa de 50\% e razão de prevalência de 2,67 (IC 1,51-4,71; $\mathrm{p}=0,0083)$. A HAS teve frequência relativa de 33,56\% e razão de prevalência de 0,76 (IC 0,54-1,07; p=0,16); a DLP apresentou frequência relativa de $42,30 \%$ e razão de prevalência de 1,12 (IC 0,81-1,53; p=0,58); e a história prévia de AVC, frequência relativa de $70 \%$ e razão de prevalência de 1,82 (IC 1,22-2,71; $\mathrm{p}=0,06$ ).

A figura 1 representa o número absoluto de pacientes com RDNP de leve a moderada, RDNP grave, RDP e EM de cada fator de risco com valor estatisticamente significativo encontrado.

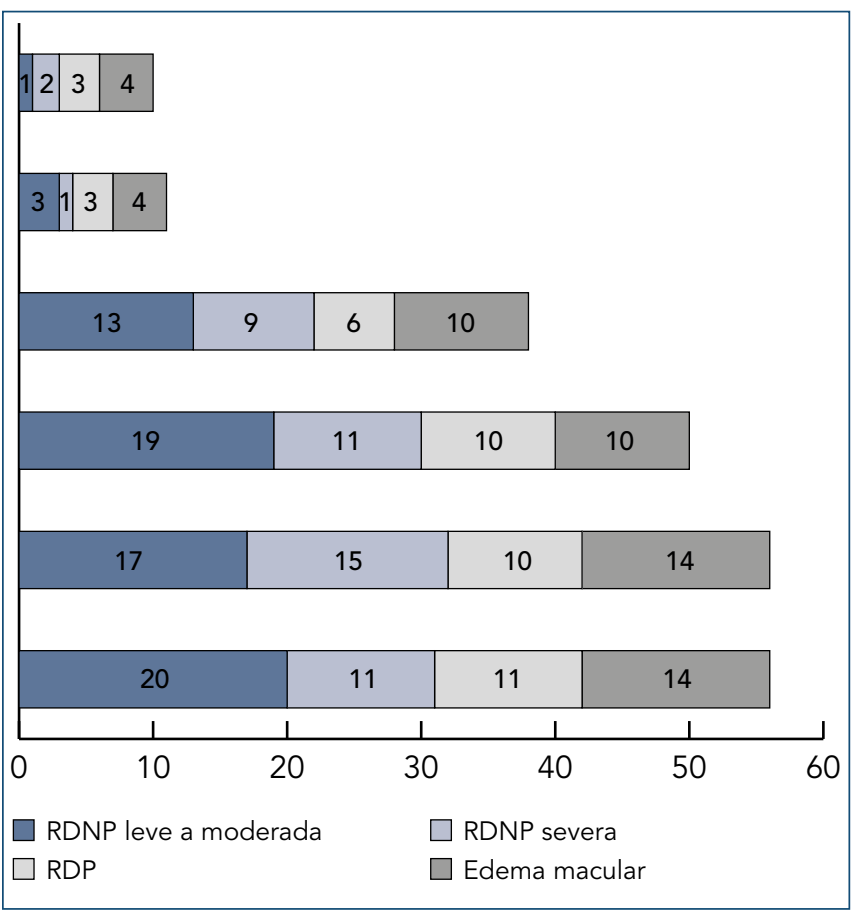

RNDP: Retinopatia Diabética Não Proliferativa; RDP: Retinopatia Diabética Proliferativa.

Figura 1. Número absoluto de pacientes que apresentaram Retinopatia Diabética de acordo com o Fator de Risco.

\section{DISCUSSÃO}

O estudo foi realizado com 219 pacientes diabéticos atendidos por demanda espontânea, dos quais 70 (31,96\%) apresentavam RD em diferentes estágios. A prevalência foi pouco menor se comparada à encontrada em pesquisa longitudinal de 1 ano, realizada na China, com 1.008 pacientes diabéticos - 40\% da população estudada apresentava alguma forma de RD. ${ }^{(8)}$ Entretanto, em comparação a outro estudo realizado durante 4 anos na Polônia, a porcentagem de cada categoria foi significativamente maior: RDNP em 19,02\% dos 1.209 poloneses diabéticos versus 33,98\% dos pacientes avaliados nesta pesquisa; RDP em 1,16\% versus 7,76\% e, por fim, EM em 4,96\% versus 9,59\%. ${ }^{\left({ }^{9}\right)}$ Ressaltam-se, portanto, o grave acometimento ocular na população goiana com DM e a necessidade de melhor assistência à essa afecção. 
A análise dos dados do presente estudo permitiu observar que a RD se mostrou mais prevalente em pacientes situados na faixa etária de 50 a 70 anos, assim como entre aqueles com mais de 10 anos de doença. Em 2009, o Wisconsin Epidemiologic Study of Diabetic Retinopathy, em um estudo de coorte de 25 anos com pacientes com DM tipo 1, demonstrou que a RD é resultado da progressão de uma retinopatia basal existente em pacientes com DM. Nesse mesmo estudo, pacientes com DM tipo 2 sem retinopatia apresentaram 54\% a menos de progressão para RD quando comparados com pacientes que já apresentavam RDNP de base no início do estudo. ${ }^{(3,10)}$ Por conseguinte, pacientes da faixa etária mencionada sugerem, por sua idade, mais tempo de desenvolvimento de doença - seja esta DM tipo 1 ou 2 -, o que configura maior risco para a progressão e o aparecimento de RD.

De acordo com o Censo de 2010, ${ }^{(11)}$ a parcela mais significativa da população de Goiânia apresentava idade entre 20 e 30 anos. Seguindo a tendência do país de aumento da expectativa de vida, é de se esperar um acréscimo considerável da população em faixa de risco para RD para as próximas décadas. Faz-se necessário, então, já no momento presente, que as políticas públicas atuem com veemência em busca do diagnóstico precoce do diabetes entre os indivíduos adultos, a fim de evitar um aumento abrupto de pacientes com perda visual irreversível ainda em idade ativa, além dos gastos com procedimentos oftalmológicos que visam manter a visão residual e evitar a progressão da RD.

Constatou-se também maior prevalência de RD em pacientes com IMC acima de 40, configurando obesidade grau III. O excesso de tecido adiposo tem íntima correlação com a fisiopatologia do DM tipo 2, contribuindo para o desenvolvimento de resistência insulínica e falência de células beta no pâncreas. Assim, em um indivíduo obeso, há potencialização da resistência insulínica, o que dificulta o controle glicêmico, criando um território favorável ao desencadeamento dos eventos fisiopatológicos necessários para o estabelecimento da RD. ${ }^{(12-14)}$ No que se trata do DM tipo 1, existem, na literatura, hipóteses que estabelecem uma relação positiva entre o ganho de peso e o desenvolvimento dessa doença em crianças. ${ }^{(12,15)}$

Dentre os 219 pacientes selecionados, 31,96\% (70) apresentavam alterações retinianas compatíveis com RD. A metade correspondia à RDNP. Desse grupo de 70 pessoas, 53,4\% eram do sexo masculino, o que configurou importante fator de risco na população estudada. Tendo em vista que a prevalência de RD em uma comunidade está intimamente atrelada ao diagnóstico precoce e ao correto manejo do $\mathrm{DM},{ }^{(16)}$ a procura de indivíduos do sexo masculino por assistência médica é notadamente mais tímida quando comparada à do sexo feminino.

De acordo com a última Pesquisa Nacional de Saúde realizada pelo Instituto Brasileiro de Geografia Estatística (IBGE) em 2013, a proporção de mulheres que tiveram acesso à consulta médica foi equivalente a $78 \%$ versus 63\% do sexo masculino. ${ }^{(17)}$ Tal cenário reforça a correlação do diagnóstico tardio de DM com o desenvolvimento de $\mathrm{RD}$ em pacientes do sexo masculino, devido ao controle inadequado da doença. Essa situação pode se apresentar como potencial frustração para o paciente e o médico, uma vez que as alterações retinianas irreversíveis provocadas pelo diabetes são plausíveis de serem postergadas por meio de um bom controle glicêmico e pelo acompanhamento oftalmológico adequado.(3)

Dados do Vigitel de 2019 apontam que, na capital goiana, 52,7\% da população apresenta sobrepeso e 19,5\%, obesidade (sem definição de grau específico), tendo o sexo masculino como majoritário em ambos os grupos $58,3 \%$ e 16,7\% dos homens da capital apresentam excesso de peso e obesidade, respectivamente. ${ }^{(18)}$ Reforça-se, destarte, a carência de ações voltadas para essa fração da sociedade nessa região.

Com valor p de 0,0083, a história pregressa de IAM constituiu fator de risco para RD. O IAM evidencia o avanço da doença sobre os órgãos-alvos, visto ser decorrente de complicações vasculares consequentes dos mecanismos fisiopatológicos do DM, sendo relacionado à recorrência de eventos cardiovasculares. ${ }^{(19,20)}$ Os desdobramentos vasculares se manifestam no olho na forma de RD, evidenciando o avanço da doença sobre os órgãos-alvos e, portanto, e fazem parte da estratificação de risco cardiovascular em pacientes diabéticos. ${ }^{(21)}$

Apesar de existirem, na literatura, evidências de que um bom controle pressórico e a manutenção da pressão arterial sistólica em níveis abaixo de $144 \mathrm{mmHg}$ minimizam os danos microvasculares aos quais se associa a RD, o presente estudo não encontrou, na amostra avaliada, valores de significância estatística, quanto ao antecedente patológico de HAS. (3,22) $^{2}$

A prática de atividade física foi encontrada como fator protetor para o desenvolvimento de RD. O exercício aeróbio e o de resistência estimulam a captação de glicose em tecidos periféricos, impactando positivamente nos níveis glicêmicos e, consequentemente, no prognóstico. ${ }^{(23)}$

No que se refere à DLP e ao AVC, apesar de estarem clinicamente integrados com o $\mathrm{DM}_{1}{ }^{(20,24,25)}$ não apresentaram correlação significativa com o desenvolvimento de RD no 
presente estudo. Igualmente, o tabagismo $(\mathrm{p}=0,84)$ não se mostrou relevante, apesar de ser, assim como a RD, um indicador de risco cardiovascular em pacientes diabéticos. ${ }^{(21)}$ Destaca-se que, em Goiânia, 8,7\% dos habitantes se declaram fumantes, um percentual consideravelmente inferior ao de outros municípios, como Porto Alegre (14,6\%), São Paulo (13,5\%) e Distrito Federal (12\%). . $^{(18)}$

Quanto ao uso de insulina, foi obtido valor de p=o,o0016, o que implicaria relevância estatística quanto à presença de RD. No entanto, não foi realizada análise de regressão, sendo, então, criado um viés de confundimento, uma vez que um paciente adulto com DM tipo 1 é insulinodependente e apresenta maior tempo de doença, podendo ter, então, RD devido a esse último fator mencionado. ${ }^{\left({ }^{10}\right)}$ Cabe destacar que a frequência relativa de pacientes com DM tipo 1 encontrada foi de 52,1\%, sem distinção específica da faixa etária abrangida. Já o paciente com DM tipo 2 pode ser insulinodependente por não ter bom controle glicêmico ou por já ter muito tempo de doença também, podendo ter $\mathrm{RD}$ por essas duas últimas razões. ${ }^{(3)}$

Ressalta-se ainda, como limitação desta pesquisa, a impossibilidade de tal amostra representar características geográficas fidedignas da população do estado, visto que o mutirão era voltado para pacientes diabéticos por demanda espontânea, e não para o público geral. Além disso, as informações referentes aos antecedentes patológicos, aos hábitos de vida e ao uso de medicação foram obtidas do relato dos pacientes avaliados, sem possibilidade de comprovação, o que poderia implicar em resultado de ausência de significância estatística, no que se refere à DLP, ao IAM, ao AVC, à atividade física e ao tabagismo.

\section{CONCLUSÃO}

Considerando o protagonismo da RD quando se trata de cegueira, nota-se a importância dessa doença não só no âmbito da saúde pública, mas também na esfera social e econômica. O presente estudo encontrou, na amostra estudada, alta prevalência de retinopatia diabética. Os indivíduos do sexo masculino apresentaram risco aumentado de progressão para um quadro ocular de retinopatia diabética. O risco se mostrou elevado também para aqueles com obesidade grau III, idade entre 51 a 70 anos ou mais de 10 anos de progressão do diabetes mellitus e com infarto agudo do miocárdio prévio. A prática de atividade física mostrou-se como fator relacionado à ausência de retinopatia diabética. Quanto ao tabagismo, à dislipidemia e ao acidente vascular cerebral, destaca-se a limitação desta pesquisa quanto à obtenção de tais dados, que se deram apenas por meio do relato dos pacientes, não permitindo comprovação, o que pode ter interferido no resultado obtido.

Estudos populacionais ao longo dos anos comprovaram a variabilidade geográfica na prevalência da retinopatia diabética justificada pela diferente exposição aos fatores de risco. Dentro de tal conjuntura, ressalta-se o quão fundamental é o conhecimento das características regionais, de modo a orientar as políticas de saúde pública, permitindo atuar com impacto na redução das estatísticas de cegueira evitável.

\section{AGRADECIMENTOS}

A toda equipe da Fundação Banco de Olhos de Goiás, que possibilitou a execução deste trabalho, e a todos os envolvidos no Mutirão do Diabetes de 2018, como acadêmicos de medicina, endocrinologistas, residentes em Oftalmologia, fellows de retina, retinólogos e demais profissionais.

\section{REFERÊNCIAS}

1. Sociedade Brasileira de Diabetes (SBD). Posicionamento Oficial SBD n.1/2019. Conduta terapêutica no diabetes tipo 2: algoritmo SBD 2019. São Paulo: SBD; 2019. [citado 2021 Mar 18]. Disponível em: https://www. diabetes.org.br/publico/images/pdf/sbd_dm2_2019_2.pdf

2. Brasil. Ministério da Saúde. Vigitel Brasil 2018. Vigilância de fatores de risco e proteção para doenças crônicas por inquérito telefônico: estimativas sobre frequência e distribuição sociodemográfica de fatores de risco e proteção para doenças crônicas nas capitais dos 26 estados brasileiros e no Distrito Federal em 2018. Brasília, DF: Ministério da Saúde; 2019. [citado 2021 Mar 18]. Disponível em: https://portalarquivos2.saude.gov.br/images/pdf/2019/julho/25/ vigitel-brasil-2018.pdf

3. Solomon SD, Chew E, Duh EJ, Sobrin L, Sun JK, VanderBeek BL, et al. Diabetic retinopathy: a position statement by the American Diabetes Association. Diabetes Care. 2017;40(3):412-8.

4. Ávila M, Alves MR, Nishi M. As condições de saúde ocular no Brasil. São Paulo: CBO; 2015. [citado 2021 Mar 18]. Disponível em: http://www.cbo. net.br/novo/publicacoes/Condicoes_saude_ocular_IV.pdf

5. Jin G, Xiao W, Ding X, Xu X, An L, Congdon N, et al. Prevalence of and risk factors for diabetic retinopathy in a rural Chinese population: the Yangxi eye study. Invest Ophthalmol Vis Sci. 2018;59(12):5067-73.

6. Associação Brasileira para o Estudo da Obesidade e da Síndrome Metabólica. Diretrizes brasileiras de obesidade 2009/2010. 3ª . ed. Itapevi: AC Farmacêutica; 2009. [citado 2021 Mar 18]. Disponível em: http:// www.saude.df.gov.br/wp-conteudo/uploads/2018/08/2009_DIRETRIZES_ BRASILEIRAS_DE_OBESIDADE.pdf

7. Wu L, Fernandez-Loaiza P, Sauma J, Hernandez-Bogantes E, Masis M. Classification of diabetic retinopathy and diabetic macular edema. World J Diabetes. 2013;4(6):290-4.

8. Yin L, Zhang D, Ren Q, Su X, Sun Z. Prevalence and risk factors of diabetic retinopathy in diabetic patients: a community based cross-sectional study. Medicine (Baltimore). 2020;99(9):e19236.

9. Matuszewski W, Baranowska-Jurkun A, Stefanowicz-Rutkowska MM, Modzelewski R, Pieczyński J, Bandurska-Stankiewicz E. Prevalence of diabetic retinopathy in type 1 and type 2 diabetes mellitus patients in North-East Poland. Medicina (Kaunas). 2020;56(4):164.

10. Klein R, Knudtson MD, Lee KE, Gangnon R, Klein BE. The Wisconsin Epidemiologic Study of Diabetic Retinopathy XXIII: the twenty-fiveyear incidence of macular edema in persons with type 1 diabetes. Ophthalmology. 2009;116(3):497-503. 
11. Instituto Brasileiro de Geografia e Estatística (IBGE). Censo 2010. Rio de Janeiro: IBGE; 2010. [citado 2021 Mar 18]. Disponível em: https:// censo2010.ibge.gov.br/

12. Al-Goblan AS, Al-Alfi MA, Khan MZ. Mechanism linking diabetes mellitus and obesity. Diabetes Metab Syndr Obes. 2014;7:587-91.

13. Wang W, Lo AC. Diabetic retinopathy: pathophysiology and treatments. Int J Mol Sci. 2018;19(6):1816

14. Rübsam A, Parikh S, Fort PE. Role of inflammation in diabetic retinopathy. Int J Mol Sci. 2018;19(4):942.

15. Wilkin TJ. The accelerator hypothesis: weight gain as the missing link between Type I and Type II diabetes. Diabetologia. 2001;44(7):914-22.

16. International Council of Ophthalmology (ICO). ICO Guidelines for Diabetic Eye Care. Updated 2017. Geneva: ICO; 2017. [citado 2021 Mar 18]. Disponível em: http://www.icoph.org/downloads/ICOGuidelinesforDiabeticEyeCare.pdf

17. Instituto Brasileiro de Geografia e Estatística (IBGE). Pesquisa nacional de saúde 2013: acesso e utilização dos serviços de saúde, acidentes e violências - Brasil, grandes regiões e unidades da federação. Rio de Janeiro: IBGE; 2015.

18. Brasil. Ministério da Saúde. Vigitel Brasil 2019. Vigilância de fatores de risco e proteção para doenças crônicas por inquérito telefônico: estimativas sobre frequência e distribuição sociodemográfica de fatores de risco e proteção para doenças crônicas nas capitais dos 26 estados brasileiros e no Distrito Federal em 2019. Brasília, DF: Ministério da Saúde; 2020. [citado 2021 Mar 18]. Disponível em: http://bvsms.saude.gov.br/ bvs/publicacoes/vigitel_brasil_2019_vigilancia_fatores_risco.pdf
19. Bianco HT, Izar MC, Fonseca HA, Póvoa RM, Saraiva JF, Forti A, et al. Relevância de lesões em órgãos-alvo como preditores de mortalidade em pacientes com diabetes. Arq Bras Cardiol. 2014;103(4):272-81.

20. Triches C, Schaan BD, Gross JL, Azevedo MJ. Complicações macrovasculares do diabetes melito: peculiaridades clínicas, de diagnóstico e manejo. Arq Bras Endocrinol Metabol. 2009;53(6):698708 .

21. Bertoluci MC, Moreira RO, Faludi A, Izar MC, Schaan BD, Valerio CM, et al Brazilian guidelines on prevention of cardiovascular disease in patients with diabetes: a position statement from the Brazilian Diabetes Society (SBD), the Brazilian Cardiology Society (SBC) and the Brazilian Endocrinology and Metabolism Society (SBEM). Diabetol Metab Syndr. 2017;9(1):53.

22. King P, Peacock I, Donnelly R. The UK prospective diabetes study (UKPDS): clinical and therapeutic implications for type 2 diabetes. $\mathrm{Br} \mathrm{J}$ Clin Pharmacol. 1999;48(5):643-8.

23. Sociedade Brasileira de Diabetes (SBD). Posicionamento Oficial n. 04/2015. Atividade física e diabetes: a prática segura de atividades desportivas. São Paulo: SBD; 2015. [citado 2021 Mar 18]. Disponível em: https://www. diabetes.org.br/profissionais/images/2017/posicionamento-4.pdf

24. Faludi AA, Izar MC, Saraiva JF, Chacra AP, Bianco HT, Afiune Neto A et al. Atualização da Diretriz Brasileira de Dislipidemias e Prevenção da Aterosclerose - 2017. Arq Bras Cardiol. 2017;109(2 Supl.1):1-76.

25. Schaan BD, Portal VL. Fisiopatologia da doença cardiovascular no diabetes. Rev Soc Cardiol Rio Grande do Sul. 2004;3:1-5. 